\title{
Efficacy of Pooled Serum Internal Quality Control in Comparison with Commercial Internal Quality Control in Clinical Biochemistry Laboratory
}

\author{
Sweta Kulkarni ${ }^{1} \quad$ Shema Alain Pierre ${ }^{1} \quad$ Ramachandran Kaliaperumal $^{1}$ \\ ${ }^{1}$ Department of Biochemistry, Mahatma Gandhi Medical College \\ and Research Institute, Sri Balaji Vidyapeeth (Deemed to be \\ University), Pondicherry, India \\ Address for correspondence Sweta Kulkarni, MBBS, MD, \\ Department of Biochemistry, Mahatma Gandhi Medical College and \\ Research Institute, Sri Balaji Vidyapeeth (Deemed to be University), \\ Pondicherry 607402, India (e-mail: shwetakulkarni82@gmail.com).
}

J Lab Physicians:2020;12:191-195

\begin{abstract}
Keywords

- internal quality control

- pooled serum

- mean

- standard deviation

$-\mathrm{CV} \%$

\section{Introduction}

Quality control (QC) is an essential component in every clinical laboratory, as it maintains the excellence of laboratory standards, supplementing proper disease diagnosis and patient care and resulting in overall strengthening of the healthcare system. 1 The increasing automation in clinical laboratories has increased the requirement of QC material for monitoring of laboratory performance. ${ }^{2}$
\end{abstract}

Introduction With increasing automation in clinical laboratories, the requirements for quality control (QC) material have greatly increased in order to monitor performance. The constant use of commercial control material is not economically feasible for many countries because of nonavailability or the high-cost of those materials. Therefore, preparation and use of in-house QC serum will be a very cost-effective measure with respect to laboratory needs.

Materials and Methods In-house internal quality control from leftover serum samples of master health checkup subjects, which have been screened negative for HIV, $\mathrm{HCV}$ and HBsAg antibodies was pooled in a glass jar with ethanediol as preservative and kept in deep freezer at $-20^{\circ} \mathrm{C}$. From the pooled serum, 100 microliter thirty aliquots were prepared. Every day along with commercial internal QC (IQC), one aliquot of pooled serum was analyzed for 30 days for the following parameters: plasma glucose, blood urea, serum creatinine, total cholesterol, triglycerides (TGL), high-density lipoprotein, calcium, total protein, albumin, total bilirubin, AST, ALT, ALP, amylase. After getting 30 values for each parameter, mean, standard deviation (SD) and CV\% were calculated for both IQC commercial sample and pooled serum sample.

Results The mean, SD, and CV\% of glucose, cholesterol, TGL, calcium, alanine aminotransaminase (ALT), aspartate aminotransferase (AST), amylase, and alkaline phosphatase (ALP) were statistically significant between pooled serum and commercial QC. Conclusion In-house QC prepared from pooled serum is better than commercial internal QC. The biochemical parameters were stable in pooled serum due to less matrix effect; also, variation was less in pooled serum IQC.
DOI https://doi.org/ $10.1055 / \mathrm{s}-0040-1721151$ ISSN 0974-2727.
The constant use of commercial QC material is not economically feasible for many countries because of nonavailability or the high-cost of these materials. The disadvantages of commercial material are vial-to-vial variation in the concentration of their constituents; besides, no matter how carefully the vials are filled, reconstitution of material can introduce additional errors. ${ }^{3}$ As per NABL guidelines, the frequency of running QC is at least twice per day and continuous use of commercial QC materials is costlier.

(c) 2020. The Indian Association of Laboratory Physicians.

This is an open access article published by Thieme under the terms of the Creative Commons Attribution-NonDerivative-NonCommercial-License, permitting copying and reproduction so long as the original work is given appropriate credit. Contents may not be used for commercial purposes, or adapted, remixed, transformed or built upon. (https://creativecommons.org/licenses/by-nc-nd/4.0/)

Thieme Medical and Scientific Publishers Pvt. Ltd., A-12, 2nd Floor, Sector 2, Noida-201301 UP, India 
The quality assurance system in clinical chemistry allows for identification of errors and control actions to correct them. Laboratory errors can be classified into preanalytical, analytical, and postanalytical. While preanalytical and postanalytical errors are difficult to identify, the analytical variability (both imprecision and inaccuracy) can be monitored with internal quality control (IQC) programs and external quality assessment (EQA) schemes. ${ }^{4}$ The purpose of IQC is mainly to verify the reliability of laboratory estimates with time.

IQC programs are based on the use of control samples which are analyzed in each analytical series. Freeze-dried (lyophilized) and liquid preparations of commercial sera are available. 5 It is a common, but unscientific practice to buy these imported costly materials in small quantity to be used infrequently and compare the values obtained during analysis with the wide range supplied by the manufacturer. The requirement for QC sera also includes sufficient supply of QC material for 1 to 2 years from the same lot, frequent $\mathrm{QC}$ runs as per scientific analytical protocols, as well as stability of QC material over the period of intended use. Hence, there was a need to prepare liquid QC serum stabilized with ethanediol, using modification of WHO recommended protocol, by a simple process requiring routine laboratory expertise at a considerably low cost.

\section{Materials and Methods}

The present analytical study was conducted in the clinical biochemistry laboratory of a tertiary care hospital after obtaining the institute's human ethical clearance (2018/06/09). The objectives of our study were to prepare low-cost in-house QC pooled serum from leftover serum samples of healthy master health checkup subjects and compare the efficacy of pooled serum in-house QC with commercial IQC.

The leftover serum samples from all healthy master health checkup patients, which tested negative for HIV, HBsAg, and HCV antibodies, were selected for the study. The lipemic, icteric, and hemolyzed samples were excluded from the study.

Every day after analysis, the leftover serum samples $(100 \mu \mathrm{L})$ were pooled in a glass jar with $100 \mathrm{~mL}$ of ethanediol as a preservative. The same procedure was repeated every day for a period of 1 month. The pooled serum was stored at $-20^{\circ} \mathrm{C}$ in a deep freezer until further analysis. From the pooled serum, $100 \mu \mathrm{L}$ of 30 aliquots were made as in-house IQCs and kept in freezer compartment of refrigerator.

The commercial QC was in lyophilized form. It was aliquoted into 10 aliquots and stored in the freezer compartment of refrigerator. Every day along with commercial IQC, 1 aliquot of in-house QC sample was run for 30 days in the morning shift, as maximum number of samples (routine and emergency) were processed in the morning shift and 1 aliquot of commercial QC in the afternoon shift, according to NABL guidelines. Both the $\mathrm{QC}$ values were recorded and plotted in the Levey-Jennings chart, and the Westgard multirules were applied. For any outliers in commercial and in-house QC, corrective and preventive action was taken and documented. The same procedure was repeated for 3 months (January, February, and March 2019). The data was entered in a Microsoft Excel sheet. The mean, standard deviation (SD) and coefficient of variation were calculated using the formula. The $\mathrm{CV} \%$ of study parameters in pooled serum was compared with commercial QC CV\%. CLIA guidelines for acceptable performance was also verified for acceptable $\mathrm{CV} \%$.

Statistical analysis was done using SPSS 17 Med Calc 9.0.1 version. Descriptive and inferential statistical analysis was performed in the present study. Results of continuous measurements were presented as mean $\pm \mathrm{SD}$ (minimum maximum), and results on categorical measurements were presented in number (\%). Statistical significance was assessed at a $5 \%$ level of significance. $p<0.05$ was considered as statistically significant. Student " $t$ " test was applied to find the difference between pooled serum IQC and commercial IQC.

\section{Results}

The mean, SD, and CV\% of both in-house and commercial IQC were calculated using the formula for 3 months (January, February, and March 2019).

There was statistically significant difference between biochemical parameters glucose, cholesterol, TGL, calcium, liver enzymes AST and ALT in in-house QC when compared with commercial IQC ( - Table 1).

In the month of February there was statistically significant difference between the in-house and commercial internal quality control parameters like the blood glucose, urea, creatinine, cholesterol, triglycerides, alanine aminotransaminase (ALT), aspartate aminotransferase (AST), and amylase $(p<0.05)$. Other biochemical parameters like urea, alkaline phosphatase, albumin, total protein, and total bilirubin were not statistically significant (-Table 2 ).

In the month of March there was statistically significant difference between the in-house and commercial internal quality control parameters like the blood glucose, urea, creatinine, cholesterol, triglycerides (TGL), ALT, AST, and amylase $(p<0.05)$. Other biochemical parameters like urea, alkaline phosphatase, albumin, total protein, and total bilirubin were not statistically significant ( - Table $\mathbf{3}$ ).

\section{Discussion}

Tertiary care hospital laboratories receive over a 1000 samples a day. These laboratories face many challenges, including equipment breakdown, and lack of standard reagents and quality control material, which can prevent same-day processing of samples. Maintenance of daily accuracy is an indispensable part of any healthcare clinical laboratory. The total analytical error (TAE) in a clinical laboratory depends upon the maintenance of both precision and accuracy, the two watch words for the total reliability of laboratory results.

QC material is important for daily checking of analytical procedures to ensure reliable laboratory results from patients' samples. However, many developing countries are disadvantaged by unavailability and high-cost of commercial 
Table 1 Comparison of in-house and commercial IQC for January 2018

\begin{tabular}{|c|c|c|c|c|c|}
\hline \multirow[t]{2}{*}{ Parameters } & \multicolumn{2}{|c|}{ Mean \pm SD } & \multicolumn{2}{|c|}{ CV\% } & \multirow[t]{2}{*}{$p$-Value } \\
\hline & Commercial QC & In-house QC & Commercial QC & In-house QC & \\
\hline Glucose (mg/dl) & $81.51 \pm 5.82$ & $105.03 \pm 5.38$ & 7.14 & 5.12 & $0.001^{\mathrm{a}}$ \\
\hline Blood urea (mg/dl) & $32.75 \pm 1.28$ & $24.52 \pm 1.51$ & 3.92 & 6.16 & 0.39 \\
\hline Creatinine (mg/dl) & $2.09 \pm 0.19$ & $1.79 \pm 0.14$ & 9.28 & 7.32 & $0.009^{\mathrm{a}}$ \\
\hline Cholesterol (mg/dl) & $256.72 \pm 8.8$ & $217.95 \pm 5.22$ & 3.43 & 2.39 & $0.001^{\mathrm{a}}$ \\
\hline TGL (mg/dl) & $175.95 \pm 6.1$ & $135.29 \pm 2.5$ & 3.47 & 1.84 & $0.001^{\mathrm{a}}$ \\
\hline $\mathrm{HDL}(\mathrm{mg} / \mathrm{dl})$ & $61.54 \pm 1.44$ & $54.15 \pm 1.09$ & 2.35 & 2.02 & $0.004^{\mathrm{a}}$ \\
\hline Calcium (mg/dl) & $9.45 \pm 0.61$ & $7.59 \pm 0.37$ & 6.46 & 4.91 & $0.001^{\mathrm{a}}$ \\
\hline Total bilirubin (mg/dl) & $1.2 \pm 0.4$ & $0.88 \pm 0.06$ & 5.45 & 6.86 & 0.34 \\
\hline Direct bilirubin (mg/dl) & $0.23 \pm 0.01$ & $0.22 \pm 0.01$ & 5.69 & 6.44 & 0.234 \\
\hline $\operatorname{ALT}(\mathrm{U} / \mathrm{L})$ & $30.18 \pm 1.97$ & $23.71 \pm 1.1$ & 6.53 & 4.67 & $0.001^{\mathrm{a}}$ \\
\hline AST (U/L) & $41.03 \pm 1.82$ & $30.2 \pm 0.88$ & 4.44 & 2.91 & $0.001^{\mathrm{a}}$ \\
\hline $\operatorname{ALP}(\mathrm{U} / \mathrm{L})$ & $106 \pm 28.38$ & $97.39 \pm 12.04$ & 26.7 & 12.36 & 0.146 \\
\hline Total protein (g/dl) & $6.8 \pm 0.42$ & $5.8 \pm 0.58$ & 4.56 & 4.84 & 0.267 \\
\hline $\operatorname{ALB}(\mathrm{g} / \mathrm{dl})$ & $4.2 \pm 0.23$ & $4.14 \pm 0.23$ & 5.69 & 5.66 & 0.344 \\
\hline Amylase (IU/L) & $62.46 \pm 2.12$ & $106.3 \pm 28.3$ & 5.88 & 3.4 & $0.001^{\mathrm{a}}$ \\
\hline
\end{tabular}

Abbreviations: ALB, albumin; ALP, alkaline phosphatase; ALT, alanine aminotransaminase; AST, aspartate aminotransferase; HDL, high-density lipoprotein; IQC, internal quality control; SD, standard deviation; TGL, triglycerides. ${ }^{a} p<0.05$.

Table 2 Comparison of in-house and commercial IQC for February 2018

\begin{tabular}{|c|c|c|c|c|c|}
\hline \multirow[t]{2}{*}{ Parameters } & \multicolumn{2}{|c|}{ Mean \pm SD } & \multicolumn{2}{|c|}{ CV\% } & \multirow[t]{2}{*}{$p$-Value } \\
\hline & Commercial QC & In-house QC & Commercial QC & In-house QC & \\
\hline Glucose (mg/dl) & $83.79 \pm 5.78$ & $105.03 \pm 5.12$ & 6.9 & 4.87 & $0.001^{\mathrm{a}}$ \\
\hline Blood urea (mg/dl) & $32.87 \pm 1.26$ & $24.53 \pm 1.12$ & 3.84 & 4.59 & 0.0892 \\
\hline Creatinine (mg/dl) & $2.1 \pm 0.19$ & $1.93 \pm 0.11$ & 9.11 & 5.8 & $0.004^{\mathrm{a}}$ \\
\hline Cholesterol (mg/dl) & $259.47 \pm 9.44$ & $215.26 \pm 22.76$ & 3.64 & 1.57 & $0.001^{\mathrm{a}}$ \\
\hline TGL (mg/dl) & $176.43 \pm 6.54$ & $135.84 \pm 2.21$ & 3.71 & 1.63 & $0.001^{\mathrm{a}}$ \\
\hline $\mathrm{HDL}(\mathrm{mg} / \mathrm{dl})$ & $61.98 \pm 1.26$ & $54.68 \pm 0.86$ & 2.04 & 1.57 & $0.001^{\mathrm{a}}$ \\
\hline Calcium (mg/dl) & $9.56 \pm 0.11$ & $9.47 \pm 0.09$ & 1.22 & 1.05 & $0.003^{\mathrm{a}}$ \\
\hline Total bilirubin (mg/dl) & $1.4 \pm 0.2$ & $0.68 \pm 0.03$ & 5.32 & 6.54 & 0.32 \\
\hline Direct bilirubin (mg/dl) & $0.24 \pm 0.01$ & $0.19 \pm 0.02$ & 5.21 & 12.27 & 0.361 \\
\hline $\operatorname{ALT}(\mathrm{U} / \mathrm{L})$ & $30.87 \pm 1.62$ & $23.9 \pm 0.59$ & 5.25 & 2.48 & $0.001^{\mathrm{a}}$ \\
\hline AST (U/L) & $40.56 \pm 1.91$ & $30.4 \pm 0.92$ & 4.71 & 3.03 & $0.001^{\mathrm{a}}$ \\
\hline $\operatorname{ALP}(\mathrm{U} / \mathrm{L})$ & $105.04 \pm 29.22$ & $102.51 \pm 12.22$ & 27.81 & 11.92 & 0.697 \\
\hline Total protein (g/dl) & $6.8 \pm 0.42$ & $5.8 \pm 0.58$ & 4.56 & 4.84 & 0.267 \\
\hline ALB (g/dl) & $4.16 \pm 0.26$ & $4.1 \pm 0.18$ & 6.32 & 4.46 & 0.36 \\
\hline Amylase (IU/L) & $62.08 \pm 3.25$ & $61.93 \pm 2.07$ & 5.24 & 3.348 & $0.032^{\mathrm{a}}$ \\
\hline
\end{tabular}

Abbreviations: ALB, albumin; ALP, alkaline phosphatase; ALT, alanine aminotransaminase; AST, aspartate aminotransferase; HDL, high-density lipoprotein; IQC, internal quality control; SD, standard deviation; TGL, triglycerides. ${ }^{\mathrm{a}} \mathrm{p}<0.05$.

QC materials. Therefore, in-house pooled human serum will be a very cost effective measure with regard to laboratory needs. The control material prepared should simulate fresh human serum in terms of matrix and concentration of analytes. It must be sufficiently homogenous, stable, and noninfective. There are two main sources of QC materials, animal and human. The animal serum is disadvantageous due to matrix effect which requires supplementation with enzymes and other constituents. Therefore, human serum is suitable for preparing control materials in clinical chemistry. ${ }^{6}$ Three types of QC materials can be prepared: frozen serum, stabilized liquid serum, and lyophilized or freeze dried serum. 
Table 3 Comparison of in-house and commercial IQC for March 2018

\begin{tabular}{|c|c|c|c|c|c|}
\hline \multirow[t]{2}{*}{ Parameters } & \multicolumn{2}{|c|}{ Mean \pm SD } & \multicolumn{2}{|c|}{ CV\% } & \multirow[t]{2}{*}{$p$-Value } \\
\hline & In-house QC & Commercial QC & Commercial QC & In-house QC & \\
\hline Glucose (mg/dl) & $106.16 \pm 2.60$ & $82.53 \pm 3.62$ & 4.61 & 2.33 & $0.001^{\mathrm{a}}$ \\
\hline Blood urea (mg/dl) & $24.68 \pm 1.47$ & $32.8 \pm 1.27$ & 3.84 & 5.98 & 0.096 \\
\hline Creatinine (mg/dl) & $2.01 \pm 0.17$ & $2.03 \pm 0.10$ & 8.40 & 5.36 & $0.0071^{a}$ \\
\hline Cholesterol (mg/dl) & $209.13 \pm 4.82$ & $252.76 \pm 0.09$ & 2.98 & 2.30 & $0.005^{\mathrm{a}}$ \\
\hline TGL (mg/dl) & $133.29 \pm 2.39$ & $172.06 \pm 5.67$ & 3.30 & 1.79 & $0.001^{\mathrm{a}}$ \\
\hline $\mathrm{HDL}(\mathrm{mg} / \mathrm{dL})$ & $53.54 \pm 1.84$ & $61.01 \pm 2.71$ & 4.45 & 3.45 & $0.001^{\mathrm{a}}$ \\
\hline Calcium (mg/dl) & $7.59 \pm 0.34$ & $9.24 \pm 0.76$ & 8.22 & 4.52 & $0.001^{\mathrm{a}}$ \\
\hline Total bilirubin (mg/dl) & $1.2 \pm 0.20$ & $0.68 \pm 0.03$ & 5.32 & 6.54 & 0.32 \\
\hline Direct bilirubin $(\mathrm{mg} / \mathrm{dl})$ & $0.19 \pm 0.01$ & $0.23 \pm 0.01$ & 6.16 & 8.13 & 0.350 \\
\hline $\operatorname{ALT}(\mathrm{U} / \mathrm{L})$ & $23.6 \pm 1.55$ & $27.84 \pm 2.57$ & 9.23 & 6.60 & $0.001^{\mathrm{a}}$ \\
\hline AST (U/L) & $29.95 \pm 0.72$ & $41.31 \pm 1.67$ & 4.05 & 2.43 & $0.001^{\mathrm{a}}$ \\
\hline $\operatorname{ALP}(U / L)$ & $93.53 \pm 9.98$ & $99.46 \pm 37.90$ & 38.11 & 10.67 & 4.476 \\
\hline Total protein (g/dl) & $6.2 \pm 0.22$ & $5.1 \pm 0.53$ & 4.31 & 4.64 & 0.267 \\
\hline $\operatorname{ALB}(\mathrm{g} / \mathrm{dl})$ & $4.13 \pm 0.20$ & $4.25 \pm 0.24$ & 5.72 & 4.85 & 0.360 \\
\hline Amylase (IU/L) & $62.44 \pm 1.76$ & $61.19 \pm 3.69$ & 6.03 & 2.82 & $0.03^{a}$ \\
\hline
\end{tabular}

Abbreviations: ALB, albumin; ALP, alkaline phosphatase; ALT, alanine aminotransaminase; AST, aspartate aminotransferase; HDL, high-density lipoprotein; IQC, internal quality control; SD, standard deviation; TGL, triglycerides. ${ }^{a} p<0.01$.

Frozen serum is of less useful in developing countries where there are transportation problems and where the laboratories do not have deep freezers. The chemically stabilized QC material gives rise to matrix effects and commutability problems due to interferences. Therefore, pooled serum with ethanediol as preservative is an alternate material in clinical laboratories.

Many studies have been performed in the past for the preparation of IQC. Lalani et $\mathrm{al}^{7}$ in their study showed that homemade QC from polycythemia patient is better than commercial QC. Jamtsho ${ }^{8}$ in his study proposed that stability of lyophilized QC is better than commercial QC. Bowes et $\mathrm{al}^{9}$ in their study showed that pooled serum QC is more stable and low-cost when compared with commercial QC. Khatri ${ }^{10}$ et al showed in their study by using patient sample that reliability and validity of test reports will be better when compared with commercial QC.

In our study, the in-house IQC material was prepared from left over pooled serum from healthy subjects who were recruited from master health checkup clinic after screening and consultation. Most of the study parameters were stable up to 12 weeks. However, the variation in stability of enzymes like AST, ALT and alkaline phosphatase (ALP) was noticed after 10 days of storage in commercial QC, whereas in pooled serum, enzymes were stable up to 30 days. The variation was not clinically significant. ${ }^{11}$ The variation in $\mathrm{CV} \%$ of parameters like ALP could be due to increase in activity of ALP after reconstitution of lyophilized $\mathrm{QC},{ }^{12}$ and the slight decrease in glucose levels could be due to degradation by microbial agents during the storage period over a month. The decrease in ALT level could be due to the loss of enzyme activities on prolonged storage and the interference by lactate dehydrogenase (LDH) on ALT. The increase in protein levels could be attributed to the liberation of free proteins from the glycoprotein; increased bacterial activities could produce some enzymes and other microbial products that would contribute in increasing the protein levels. Gradual decrease in total bilirubin could be due to photo degradation of bilirubin during storage. ${ }^{13}$

Another use of pooled serum is to monitor a continuous flow analysis system throughout a long series of estimations, in order to ensure that QC is maintained. Pooled serum is valuable in detecting and correcting for drift, particularly for Auto Analyzer techniques involving dialysis. Faults in the dialyzer may be detected only by protein-containing control fluids. The fact that values of pooled serum are known to the user limits its value as an independent control serum. Unconscious bias and "rounding-off" of results are inevitable. Also, pooled serum only checks precision at one level. These disadvantages, together with the fact that enzymes and bilirubin slowly deteriorate, necessitate the use of further methods of quality control. Hence, pooled serum quality control is an alternative but not a replacement for commercial QC.

\section{Conclusion}

Hence, our study emphasizes on preparation of in-house QC from pooled serum as an alternative, low-cost step to maintain regular quality planning in a clinical laboratory. The outcome of the study confirms that the pooled serum IQC is a cost-effective approach to maintain quality assurance in resource limited laboratory when compared with commercial IQCs which are costlier. The stability of most of the biochemical parameters in pooled serum with ethanediol as preservative is better, and matrix effect interferences will be less when compared with commercial IQC. 


\section{Funding}

None.

\section{Conflicts of Interest}

None.

\section{Acknowledgment}

Authors would like to acknowledge the technicians of the Clinical Biochemistry laboratory.

\section{References}

1 Kanagasabapathy AS, Swaminathan S, Selvakumar R. Quality control in clinical biochemistry. Indian J Clin Biochem 1996;11(1):17-25

2 Kenning AO, Eaton RH. Practical guidelines for the preparation of quality control sera for use in clinical chemistry. Available at: https://apps.who.int/iris/handle/10665/259944. Accessed July 11,2020

3 Ohman S. Quality control for the clinical chemistry laboratory. Qual Assur 1997;5(2):79-93

4 Swaminathan S, Seshadri MS, Srikrishna G, Selvakumar R, Kanagasabapathy AS. Ethanediol stabilised quality control serum for measurement of zinc. Clin Chim Acta 1993; 215(1):119-121

5 Middle J. External quality assurance. Ann Clin Biochem 1998;35(Pt 4) :549-550, author reply 552-553
6 Zafar MN, Syed S. Economy and quality assessment of home made clinical chemistry reagents. J Pak Med Assoc 1992; 42(4):95-97

7 Lalani R, Zafar MN, Khurshid M. Efficacy of internal and external quality control in chemical pathology. J Pak Med Assoc 1988;38(10):255-259

8 Jamtsho R. Stability of lyophilized human serum for use as quality control material in Bhutan Indian. J ClinBiochem. 2013;28(4):418-421

9 Premachandra P, Wood PL, Hill PG, Browning DM, Olazabal DA. Preparation and stability of low-cost liquid quality-control serum stabilized with ethanediol. Clin Chem 1987; 33(6):851-852

10 Khatri R, K C S, Shrestha P, Sinha JN. Implementing self sustained quality control procedures in a clinical laboratory. JNMA J Nepal Med Assoc 2013;52(189):233-237

11 Kachhawa K, Kachhawa P, Varma M, Behera R, Agrawal D, Kumar S. Study of the stability of various biochemical analytes in samples stored at different predefined storage conditions at an accredited laboratory of India. J Lab Physicians 2017; 9(1):11-15

12 Czarnetzy EM, Richeal RJ, O'Malley JA. Temperature dependent changes with time in the alkaline phosphatase activity of commercial control serum. Clin Chem 1970;16:521-522

13 Khan MAU, Khan FA. Low cost quality control human serum: method of preparation, validation of values and its comparison with the commercial control serum. J Pak Med Assoc 2004;54(7):375-378 